\title{
THE CONNECTION BETWEEN THE CEGRELL CLASSES AND COMPLIANT FUNCTIONS
}

\author{
RAFAŁ CZYŻ and PER ÅHAG*
}

\begin{abstract}
In this article the connection between the Cegrell classes and compliant functions is studied. A suitable norm is constructed which makes the compliant functions into a Banach space. As an application a characterization of the Dirichlet problem for pluriharmonic functions is achieved. Explicit examples of non-compliant functions will be constructed and a sufficient condition for compliance will be proved.
\end{abstract}

\section{Introduction}

Throughout this article let $\Omega \subseteq \mathrm{C}^{n}$ be a hyperconvex domain, i.e., a connected, open set that admits a negative plurisubharmonic exhaustion function. Furthermore it is assumed that $\Omega$ is bounded. Recall that the Perron-Bremermann envelope for a given function $f: \partial \Omega \rightarrow \mathbf{R}$ is defined by

(1) $P B_{f}(z)=\sup \left\{w(z): w \in \mathscr{P} \mathscr{S} \mathscr{H}(\Omega), \limsup _{\substack{\zeta \rightarrow \xi \\ \zeta \in \Omega}} w(\zeta) \leq f(\xi) \forall \xi \in \partial \Omega\right\}$,

where $\mathscr{P} \mathscr{S} \mathscr{H}(\Omega)$ is the class of all plurisubharmonic functions defined on $\Omega$. If $f: \partial \Omega \rightarrow \mathrm{R}$ is a continuous function, then $P B_{f} \in \mathscr{P} \mathscr{S} \mathscr{H}(\Omega)$ since a hyperconvex domain viewed as a set in $\mathrm{R}^{2 n}$ is regular to the Laplace operator. Consider the following two assertions:

$$
\begin{array}{ll}
\text { P1: } & \lim _{\substack{z \rightarrow \xi \\
z \in \Omega}}\left(P B_{f}+P B_{-f}\right)(z)=0 \quad \text { for every } \xi \in \partial \Omega, \\
\text { P2: } \quad \int_{\Omega}\left(d d^{c}\left(P B_{f}+P B_{-f}\right)\right)^{n}<+\infty,
\end{array}
$$

where $\left(d d^{c} \cdot\right)^{n}$ is the complex Monge-Ampère operator. A continuous function $f: \partial \Omega \rightarrow \mathbf{R}$ which satisfies $\mathrm{P} 1$ and $\mathrm{P} 2$ is called a compliant function. The compliant functions first arose in [3] when some of the Cegrell classes

\footnotetext{
* The first-named author was partially supported by KBN, under grant number 1 P03A 03726.
}

Received May 5, 2005. 
given by a continuous function were introduced. Let $\mathscr{C P}(\partial \Omega)$ denote the class of compliant functions defined on $\partial \Omega$. If $n=1$, then the set of compliant functions and continuous functions defined on $\partial \Omega$ coincide, therefore it will be assumed that $n \geq 2$ throughout this article. The special case when $\Omega$ is a hyperconvex product domain $\Omega=\Omega_{n_{1}} \times \cdots \times \Omega_{n_{k}}, n=n_{1}+\cdots+n_{k}, k \geq 3$, was studied in [7].

The Cegrell classes were first introduced in [3]. In [4] and [15] new classes were added to the Cegrell family. Let $\mathscr{E}_{0}(f)$ be the class of plurisubharmonic functions defined in [3] (see also Definiton 2.1). Example 2.4 shows that there exists a function $u \in \mathscr{E}_{0}(f)$ such that

$$
\int_{\Omega}\left(d d^{c} u\right)^{n}=+\infty
$$

This cannot occur if $f$ is compliant (Lemma 2.5). Let $u \in \mathscr{E}_{0}(f)$. Then $f$ is compliant if, and only if, $\left(u+P B_{-f}\right) \in \mathscr{E}_{0}(0)$. A more thorough study about this property in the different Cegrell classes will be made in Section 2. By using a convexity property of $\mathscr{E}_{0}(f)$ it is proved that a continuous function $f: \partial \Omega \rightarrow$ R is compliant if, and only if, $\mathscr{E}_{0}(f) \oplus \mathscr{E}_{0}(-f) \subseteq \mathscr{E}_{0}(0)$, where $\oplus$ is the sum of two sets (Theorem 2.7).

Let $\|\cdot\|: \mathscr{C P}(\partial \Omega) \rightarrow \mathrm{R}$ be defined by

$$
\|f\|=\|f\|_{\infty}+\left(\int_{\Omega}\left(d d^{c}\left(P B_{f}+P B_{-f}\right)\right)^{n}\right)^{\frac{1}{n}},
$$

where $\|f\|_{\infty}=\sup \{|f(\xi)|: \xi \in \partial \Omega\}$. The aim of Section 3 is to prove that $(\mathscr{C P P}(\partial \Omega),\|\cdot\|)$ is a Banach space (Theorem 3.3). Let $\mathscr{P} \mathscr{H}(\partial \Omega)$ denote those continuous functions $\partial \Omega \rightarrow \mathrm{R}$ which can be extended to a pluriharmonic function in $\Omega$. A considerable amount of results concerning the Dirichlet problem for pluriharmonic functions exist, see e.g. [1], [2], [7], [8], [9], [10], [11] and the references therein. As an application of Theorem 3.3 it is proved that $\mathscr{P} \mathscr{H}(\partial \Omega)$ is equivalent to the closed subspace, $\mathscr{C} \mathscr{P}_{0}(\partial \Omega)$, of $\mathscr{C} \mathscr{P}(\partial \Omega)$ that contains functions for which $-P B_{f}=P B_{-f}$ on $\Omega$ (Theorem 3.5).

Example 4.1 shows that it is not enough to assume that $f$ is a $C^{1}$-function to ensure that $\mathrm{P} 2$ is true, even if $\Omega$ is the unit ball. Example 4.2 shows that even if $\mathrm{P} 2$ is true and $f \in C^{\infty}(\partial \Omega)$, it may happen that $\mathrm{P} 1$ is false. If $\Omega$ is a strictly pseudoconvex domain in $\mathrm{C}^{n}$ with $C^{2}$-boundary and $f: \partial \Omega \rightarrow \mathrm{R}$ is a $C^{2}$-function, then $f$ is a compliant function (Proposition 4.3).

The authors would like to thank Jonas Andersson, Sławomir Kołodziej and Frank Wikström for many valuable comments on and suggestions for this manuscript. 


\section{The connection between the Cegrell classes and compliant functions}

In this section assume that $f: \partial \Omega \rightarrow \mathbf{R}$ is a continuous function such that

$$
\lim _{z \rightarrow \xi} P B_{f}(z)=f(\xi),
$$

for every $\xi \in \partial \Omega$, hence $P B_{f} \in \mathscr{P} \mathscr{S} \mathscr{H}(\Omega) \cap C(\bar{\Omega})$ by Walsh's theorem (see [13]). A bounded plurisubharmonic function $\varphi$ defined on $\Omega$ belongs to $\mathscr{E}_{0}\left(=\mathscr{E}_{0}(\Omega)\right)$ if $\lim _{z \rightarrow \xi} \varphi(z)=0$ for every $\xi \in \partial \Omega$ and

$$
\int_{\Omega}\left(d d^{c} \varphi\right)^{n}<+\infty
$$

The class $\mathscr{E}_{0}$ has a role similar to that of the test functions, $C_{0}^{\infty}(\Omega)$, in the theory of distributions. Before the connection between the Cegrell classes and compliant functions will be discussed the definition of the Cegrell classes given by a continuous function will be stated. For the definition of $\mathscr{F}_{p}$ and $\mathscr{E}_{p}$ see [3] and for $\mathscr{F}, \mathscr{E}$ see [4]. For further information about the Cegrell classes see e.g. [6] and the references therein.

Definition 2.1. Let $\mathscr{K} \in\left\{\mathscr{E}_{0}, \mathscr{F}_{p}, \mathscr{E}_{p}, \mathscr{F}, \mathscr{E}\right\}$ and $f: \partial \Omega \rightarrow \mathrm{R}$ a continuous function that satisfies (3). A plurisubharmonic function $u$ defined on $\Omega$ belongs to $\mathscr{K}(f)(=\mathscr{K}(\Omega, f))$ if there exists a function $\varphi \in \mathscr{K}$ such that

$$
P B_{f} \geq u \geq \varphi+P B_{f} .
$$

REMARK. Let $\mathscr{K} \in\left\{\mathscr{E}_{0}, \mathscr{F}_{p}, \mathscr{E}_{p}, \mathscr{F}, \mathscr{E}\right\}$, then $\mathscr{K}(0)=\mathscr{K}$.

Proposition 2.2. Let $\mathscr{K} \in\left\{\mathscr{E}_{0}, \mathscr{F}_{p}, \mathscr{E}_{p}, \mathscr{F}\right\}$ and let $u \in \mathscr{K}(f)$. If $f$ is a compliant function, then $\left(u+P B_{-f}\right) \in \mathscr{K}(0)$.

Proof. Let $\mathscr{K} \in\left\{\mathscr{E}_{0}, \mathscr{F}_{p}, \mathscr{E}_{p}, \mathscr{F}\right\}$ and $u \in \mathscr{K}(f)$. Definition 2.1 implies that $u \in \mathscr{P} \mathscr{S} \mathscr{H}(\Omega)$ and that there exists a function $\varphi \in \mathscr{K}$ such that $P B_{f} \geq$ $u \geq \varphi+P B_{f}$, hence

$$
0 \geq P B_{f}+P B_{-f} \geq u+P B_{-f} \geq \varphi+P B_{f}+P B_{-f} .
$$

The function $\left(\varphi+P B_{f}+P B_{-f}\right)$ belongs to $\mathscr{K}$, since $\mathscr{K}$ is a convex cone and $f$ is compliant. By (4) it follows that $\left(u+P B_{-f}\right) \in \mathscr{K}(0)$.

Remark. The converse statement of Proposition 2.2 is true for $\mathscr{E}_{0}(f)$, i.e., if $u \in \mathscr{E}_{0}(f)$ and $\left(u+P B_{-f}\right) \in \mathscr{E}_{0}(0)$, then $f$ is a compliant function. Let $\mathscr{K} \in\left\{\mathscr{F}_{p}, \mathscr{E}_{p}, \mathscr{F}\right\}$ and $u \in \mathscr{K}$. If $\left(u+P B_{-f}\right) \in \mathscr{K}(0)$, then $\left(P B_{f}+P B_{-f}\right) \in$ $\mathscr{K}(0)$. But $\left(P B_{f}+P B_{-f}\right) \in \mathscr{K}(0)$ is generally not a sufficient condition for $f$ to be compliant. 
Proposition 2.2 yields an easy method to transform questions about the Cegrell classes given by a continuous function to the classes with zero boundary values. The classes with zero boundary values is much easier to handle and therefore the question of finding a natural characterization of the compliant functions is of importance. If $u \in \mathscr{E}(f)$, then $\left(u+P B_{-f}\right)$ always belongs to $\mathscr{E}$ without the assumption that $f$ is compliant. Example 2.4 is a slightly modified version of Example 5.6 in [5] and it shows that there exists a function $u \in \mathscr{E}_{0}(f), f \in C^{\infty}(\partial \Omega)$, such that the total mass of $\left(d d^{c} u\right)^{n}$ is infinite. This cannot occur if $f$ is a compliant function (see Lemma 2.5).

Lemma 2.3. If $f, g \in \mathscr{F}$, then

$$
\left(\int_{\Omega}\left(d d^{c}(u+v)\right)^{n}\right)^{\frac{1}{n}} \leq\left(\int_{\Omega}\left(d d^{c} u\right)^{n}\right)^{\frac{1}{n}}+\left(\int_{\Omega}\left(d d^{c} v\right)^{n}\right)^{\frac{1}{n}} .
$$

Proof. See Lemma 2.5 in [5].

Example 2.4. Let $\mathrm{P}$ be the unit polydisc in $\mathrm{C}^{2}$, i.e., $P=\left\{\left(z_{1}, z_{2}\right) \in\right.$ $\left.\mathrm{C}^{2}:\left|z_{1}\right|<1,\left|z_{2}\right|<1\right\}$. Let $f: \partial P \rightarrow \mathrm{R}$ be defined by $f\left(z_{1}, z_{2}\right)=\left|z_{2}\right|^{2}$. The function $f$ is not compliant, $f \in C^{\infty}(\partial P)$ and $P B_{f}\left(z_{1}, z_{2}\right)=\left|z_{2}\right|^{2}$. For each $j \in \mathrm{N}$ define the function $\varphi_{j}: P \rightarrow \mathrm{R}$ by $\varphi_{j}(z)=\varphi_{j}\left(z_{1}, z_{2}\right)=$ $\max \left(a_{j} \log \left|z_{1}\right|, b_{j} \log \left|z_{2}\right|, c_{j}\right)$, where $a_{j}, b_{j}, c_{j} \in \mathrm{R}, a_{j}, b_{j}>0$ and $c_{j}<$ 0 . Then $\varphi_{j} \in \mathscr{P} \mathscr{S} \mathscr{H}(P) \cap C(\bar{P}), \lim _{\left(z_{1}, z_{2}\right) \rightarrow\left(\xi_{1}, \xi_{2}\right)} \varphi_{j}\left(z_{1}, z_{2}\right)=0$ for every $\left(\xi_{1}, \xi_{2}\right) \in \partial P$ and

$$
\int_{P}\left(d d^{c} \varphi_{j}\right)^{2}=(2 \pi)^{2} a_{j} b_{j}<+\infty,
$$

hence $\varphi_{j} \in \mathscr{E}_{0}$. Let $v_{k}: P \rightarrow \mathrm{R}$ be defined by $v_{k}=\sum_{j=1}^{k} \varphi_{j}$. From this definition it follows that $v_{k} \in \mathscr{E}_{0}$ and that $\left[v_{k}\right]$ is a decreasing sequence on $P$. Lemma 2.3 and (5) yields that

$$
\int_{P}\left(d d^{c} v_{k}\right)^{2} \leq\left(\sum_{j=1}^{k}\left(\int_{P}\left(d d^{c} \varphi_{j}\right)^{2}\right)^{\frac{1}{2}}\right)^{2} \leq(2 \pi)^{2}\left(\sum_{j=1}^{k}\left(a_{j} b_{j}\right)^{\frac{1}{2}}\right)^{2} .
$$

Assume that

$$
\sum_{j=1}^{\infty}\left(a_{j} b_{j}\right)^{\frac{1}{2}}<+\infty \quad \text { and } \quad \sum_{j=1}^{\infty} c_{j}>-\infty
$$

and let $v(z)=\lim _{k \rightarrow \infty} v_{k}(z)$. The construction of the function $v$ implies that $\lim _{\left(z_{1}, z_{2}\right) \rightarrow\left(\xi_{1}, \xi_{2}\right)} v\left(z_{1}, z_{2}\right)=0$ for every $\left(\xi_{1}, \xi_{2}\right) \in \partial P$. The assumptions in (7) 
imply that $v \in \mathscr{P} \mathscr{S} \mathscr{H}(P) \cap L^{\infty}(P)$ and by inequality (6) it follows that $v \in \mathscr{E}_{0}$. Let $u: P \rightarrow \mathrm{R}$ be defined by $u=v+P B_{f}$, hence $u=\left(v+\left|z_{2}\right|^{2}\right) \in \mathscr{E}_{0}(f)$. Then it follows that

$$
\begin{aligned}
\int_{P}\left(d d^{c}\left(v_{k}+\left|z_{2}\right|^{2}\right)\right)^{2} & =\int_{P}\left(d d^{c} v_{k}\right)^{2}+4 i \int_{P}\left(d d^{c} v_{k}\right) \wedge d z_{2} \wedge d \bar{z}_{2} \\
& =\int_{P}\left(d d^{c} v_{k}\right)^{2}+32 \int_{P} \frac{\partial^{2} v_{k}}{\partial z_{1} \partial \bar{z}_{1}} d V\left(\left(z_{1}, z_{2}\right)\right) \\
& =\int_{P}\left(d d^{c} v_{k}\right)^{2}+32 \int_{P} \sum_{j=1}^{k} \frac{\partial^{2} \varphi_{j}}{\partial z_{1} \partial \bar{z}_{1}} d V\left(\left(z_{1}, z_{2}\right)\right) \\
& \geq 32 \sum_{j=1}^{k} \int_{P} \frac{\partial^{2} \varphi_{j}}{\partial z_{1} \partial \bar{z}_{1}} d V\left(\left(z_{1}, z_{2}\right)\right),
\end{aligned}
$$

where $V$ is the Lebesgue measure on $C^{2}$. Let $\varepsilon>0$ be given such that $0<$ $\varepsilon<1$ and let $D(0, r)=\{z \in \mathrm{C}:|z|<r\}$. Choose $\chi_{1}, \chi_{2} \in C_{0}^{\infty}(D(0,1))$ such that $0 \leq \chi_{1}, \chi_{2} \leq 1$ and $\chi_{1}=1=\chi_{2}$ on $D(0,1-\varepsilon)$. For fixed $\left|z_{2}\right| \leq \min \left(1-\varepsilon,(1-\varepsilon)^{\frac{a_{j}}{b_{j}}}\right)$ fix, it follows that

$$
\int_{D(0,1)} \chi_{1}\left(z_{1}\right) \frac{\partial^{2} \varphi_{j}}{\partial z_{1} \partial \bar{z}_{1}} d V\left(z_{1}\right)=8 \pi a_{j} .
$$

Under the assumption that $a_{j} \geq b_{j}$ inequality (8) together with (9) yield that

$$
\begin{aligned}
\int_{P}\left(d d^{c}\left(v_{k}+\left|z_{2}\right|^{2}\right)\right)^{2} & \geq 32 \sum_{j=1}^{k} \int_{P}\left(\chi_{1}\left(z_{1}\right) \chi_{2}\left(z_{2}\right)\right) \frac{\partial^{2} \varphi_{j}}{\partial z_{1} \partial \bar{z}_{1}} d V\left(\left(z_{1}, z_{2}\right)\right) \\
& \geq c \sum_{j=1}^{k} a_{j}\left(\min \left(1-\varepsilon,(1-\varepsilon)^{\frac{a_{j}}{b_{j}}}\right)\right)^{2},
\end{aligned}
$$

where $c>0$ is a constant. Let $\varepsilon \rightarrow 0^{+}$, then (10) implies that

$$
\int_{P}\left(d d^{c}\left(v_{k}+\left|z_{2}\right|^{2}\right)\right)^{2} \geq c \sum_{j=1}^{k} a_{j} .
$$

Thus

$$
\int_{P}\left(d d^{c} u\right)^{2}=\lim _{k \rightarrow+\infty} \int_{P}\left(d d^{c}\left(v_{k}+\left|z_{2}\right|^{2}\right)\right)^{2} \geq c \sum_{j=1}^{\infty} a_{j}
$$


Let the sequences $\left[a_{j}\right],\left[b_{j}\right]$ and $\left[c_{j}\right]$ be defined by $a_{j}=1 / j, b_{j}=1 / j^{3}$ and $c_{j}=-1 / j^{2}$. Thus the assumptions (7) and $a_{j} \geq b_{j}$ are satisfied, which implies that the function defined on $P$ by

$$
u\left(z_{1}, z_{2}\right)=\sum_{j=1}^{\infty} \max \left(\frac{1}{j} \log \left|z_{1}\right|, \frac{1}{j^{3}} \log \left|z_{2}\right|,-\frac{1}{j^{2}}\right)+\left|z_{2}\right|^{2},
$$

belongs to $\mathscr{E}_{0}(f)$ and $\int_{P}\left(d d^{c} u\right)^{2}=+\infty$, by (11).

Lemma 2.5. If $f \in \mathscr{C} \mathscr{P}(\partial \Omega)$, then $\mathscr{F}(f)=\left\{u \in \mathscr{F}(f): \int_{\Omega}\left(d d^{c} u\right)^{n}<\right.$ $+\infty\}$

Proof. Let $u \in \mathscr{F}(f)$, i.e., $u \in \mathscr{P} \mathscr{S} \mathscr{H}(\Omega)$ and there exists a function $\varphi \in$ $\mathscr{F}$ such that $P B_{f} \geq u \geq \varphi+P B_{f}$. Theorem 2.1 in [4] implies that there exists a decreasing sequence $\left[\varphi_{j}\right], \varphi_{j} \in \mathscr{E}_{0}$, that converges pointwise to $\varphi$ as $j \rightarrow+\infty$. Let the sequence $\left[u_{j}\right], j \in \mathrm{N}$, be defined by $u_{j}=\max \left(u, \varphi_{j}+P B_{f}\right)$. Then the decreasing sequence $\left[u_{j}\right], u_{j} \in \mathscr{E}_{0}(f)$, converges pointwise to $u$ as $j \rightarrow+\infty$ and

$$
\begin{aligned}
\left(d d^{c}\left(\varphi_{j}+P B_{f}+P B_{-f}\right)\right)^{n} & \geq\left(d d^{c}\left(\varphi_{j}+P B_{f}\right)\right)^{n}+\left(d d^{c} P B_{-f}\right)^{n} \\
& =\left(d d^{c}\left(\varphi_{j}+P B_{f}\right)\right)^{n},
\end{aligned}
$$

since $\left(P B_{f}+P B_{-f}\right) \in \mathscr{E}_{0}(0)$. The sequence $\left[\left(\varphi_{j}+P B_{f}+P B_{-f}\right)\right]$ is decreasing and converges pointwise to $\left(\varphi+P B_{f}+P B_{-f}\right) \in \mathscr{F}$ as $j \rightarrow+\infty$. From Proposition 5.1 in [4] it follows that

$$
\lim _{j \rightarrow+\infty} \int_{\Omega}\left(d d^{c}\left(\varphi_{j}+P B_{f}+P B_{-f}\right)\right)^{n}=\int_{\Omega}\left(d d^{c}\left(\varphi+P B_{f}+P B_{-f}\right)\right)^{n},
$$

hence

$$
\sup _{j} \int_{\Omega}\left(d d^{c} u_{j}\right)^{n}<+\infty
$$

by (12). Let $\left[\phi_{s}\right], \phi_{s} \in C_{0}^{\infty}(\Omega), \phi_{s} \geq 0$, be an increasing sequence which converges pointwise to 1 on $\Omega$ as $s \rightarrow+\infty$. For $s \in \mathbf{N}$ fixed, it follows from (13) that

$$
\int_{\Omega} \phi_{s}\left(d d^{c} u\right)^{n}=\lim _{j \rightarrow+\infty} \int_{\Omega} \phi_{s}\left(d d^{c} u_{j}\right)^{n} \leq \sup _{j} \int_{\Omega}\left(d d^{c} u_{j}\right)^{n}<+\infty .
$$

Let $s \rightarrow+\infty$, this lemma then follows from (14) and the monotone convergence theorem. 
Lemma 2.6. Assume that $f, g: \partial \Omega \rightarrow \mathbf{R}$ are continuous functions such that

$$
\lim _{\substack{z \rightarrow \xi \\ z \in \Omega}} P B_{f}(z)=f(\xi) \quad \text { and } \quad \lim _{\substack{z \rightarrow \xi \\ z \in \Omega}} P B_{g}(z)=g(\xi),
$$

for every $\xi \in \partial \Omega$ and in addition assume that $f$ is a compliant function. Let $\mathscr{K} \in\left\{\mathscr{E}_{0}, \mathscr{F}_{p}, \mathscr{E}_{p}, \mathscr{F}\right\}$. If $u \in \mathscr{K}(f)$ and $v \in \mathscr{K}(g)$, then $(\alpha u+\beta v) \in$ $\mathscr{K}(\alpha f+\beta g)$, where $\alpha, \beta \in \mathrm{R}, \alpha, \beta \geq 0$.

Proof. Let $\mathscr{K} \in\left\{\mathscr{E}_{0}, \mathscr{F}_{p}, \mathscr{E}_{p}, \mathscr{F}\right\}, u \in \mathscr{K}(f)$ and $v \in \mathscr{K}(g)$. Definition 2.1 implies that $u, v \in \mathscr{P} \mathscr{S} \mathscr{H}(\Omega)$ and that there exist functions $\varphi, \psi \in \mathscr{K}$ such that $P B_{f} \geq u \geq \varphi+P B_{f}$ and $P B_{g} \geq v \geq \psi+P B_{g}$. The definition of the Perron-Bremermann envelope yields that $P B_{\beta g} \geq P B_{\alpha f+\beta g}+P B_{-\alpha f}$ and therefore it follows that

$$
\begin{aligned}
P B_{\alpha f+\beta g} \geq \alpha u+\beta v & \geq \alpha \varphi+\beta \psi+P B_{\alpha f}+P B_{\beta g} \\
& \geq \alpha \varphi+\beta \psi+P B_{\alpha f}+P B_{\alpha f+\beta g}+P B_{-\alpha f} \\
& =\alpha \varphi+\beta \psi+\alpha\left(P B_{f}+P B_{-f}\right)+P B_{\alpha f+\beta g} .
\end{aligned}
$$

Thus $(\alpha u+\beta v) \in \mathscr{K}(\alpha f+\beta g)$, since $\left(\alpha \varphi+\beta \psi+\alpha\left(P B_{f}+P B_{-f}\right)\right) \in \mathscr{K}$.

THEOREM 2.7. Let $\Omega \subseteq \mathrm{C}^{n}$ be a bounded hyperconvex domain and let $f: \Omega \rightarrow \mathrm{R}$ be a continuous function. The function $f$ is compliant if, and only if,

$$
\mathscr{E}_{0}(f) \oplus \mathscr{E}_{0}(-f) \subseteq \mathscr{E}_{0}(0),
$$

where $\oplus$ is the sum of two sets. Moreover, equality holds in (15) if, and only if, $P B_{f}$ is pluriharmonic on $\Omega$ and continuous on $\bar{\Omega}$.

Proof. Assume that $f$ is a compliant, hence $-f$ is compliant. Let $u \in$ $\mathscr{E}_{0}(f)$ and $v \in \mathscr{E}_{0}(-f)$. Lemma 2.6 implies that $(u+v) \in \mathscr{E}_{0}(f+(-f))=$ $\mathscr{E}_{0}(0)$. The converse follows immediately since $P B_{f} \in \mathscr{E}_{0}(f)$ and $P B_{-f} \in$ $\mathscr{E}_{0}(-f)$. For the second statement first assume that equality holds in (15). Then there exist $u \in \mathscr{E}_{0}(f)$ and $v \in \mathscr{E}_{0}(-f)$ such that $u+v=0$, hence $u$ is pluriharmonic on $\Omega$ and $u=P B_{f}$. Walsh's theorem concludes that $P B_{f}$ is continuous on $\bar{\Omega}$. This proof ends with noticing that if $P B_{f}$ is pluriharmonic on $\Omega$ and continuous on $\bar{\Omega}$, then $P B_{-f}=P B_{f}$ and for $u \in \mathscr{E}_{0}$ it follows that $\left(u+P B_{f}\right) \in \mathscr{E}_{0}(f)$ and $u=\left(u+P B_{f}\right)+P B_{-f}$.

Corollary 2.8 is a direct consequence of Theorem 2.7 and Lemma 2.5.

Corollary 2.8. If $f \in \mathscr{C P}(\partial \Omega)$ is a compliant function, then $\mathscr{E}_{0}(f)=$ $\left\{u \in \mathscr{E}_{0}(f): \int_{\Omega}\left(d d^{c} u\right)^{n}<+\infty\right\}$ and $\mathscr{E}_{0}(-f)=\left\{v \in \mathscr{E}_{0}(-f): \int_{\Omega}\left(d d^{c} v\right)^{n}<\right.$ $+\infty\}$. 


\section{The compliant functions as a Banach space}

For convenience let $u$ be the operator defined on a function $f: \partial \Omega \rightarrow$ R by $u(f)=P B_{f}+P B_{-f}$. Then it immediately from (1) that if $f, g: \partial \Omega \rightarrow \mathbf{R}$ are two functions and $\alpha, \beta \in \mathrm{R}$, then

$$
0 \geq u(\alpha f+\beta g) \geq|\alpha| u(f)+|\beta| u(g) .
$$

This inequality together with the fact that $\mathscr{E}_{0}$ is a convex cone yields Proposition 3.1.

Proposition 3.1. Let $\Omega \subseteq \mathrm{C}^{n}$ be a bounded hyperconvex domain. The set of all compliant functions is a linear subspace of the real vector space containing the real-valued continuous functions defined on $\partial \Omega$.

Lemma 3.2. If $f, g \in \mathscr{C} \mathscr{P}(\partial \Omega)$, then $|u(f)(z)-u(g)(z)| \leq 2\|f-g\|_{\infty}$ for all $z \in \Omega$.

Proof. The definition of the Perron-Bremermann envelope implies that $P B_{f-g} \leq P B_{f}-P B_{g}$ and $P B_{f}-P B_{g} \leq-P B_{g-f}$ and from this it follows that

$$
\min _{\partial \Omega}(g-f) \leq P B_{f-g} \leq P B_{f}-P B_{g} \leq-P B_{g-f} \leq-\min _{\partial \Omega}(f-g),
$$

hence $\left|P B_{f}-P B_{g}\right| \leq\|f-g\|_{\infty}$ and therefore

$$
|u(f)(z)-u(g)(z)|=\left|P B_{f}+P B_{-f}-P B_{g}-P B_{-g}\right| \leq 2\|f-g\|_{\infty},
$$

which concludes the proof.

Theorem 3.3. If $\|\cdot\|$ is defined by (2), then $(\mathscr{C} \mathscr{P}(\partial \Omega),\|\cdot\|)$ is a Banach space.

Proof. If $f$ is identically 0 , then it follows from (2) that $\|f\|=0$. Moreover, if $\|f\|=0$, then $\|f\|_{\infty}=0$. Hence $f=0$. Let $t \in \mathrm{R}$, then

$$
\begin{aligned}
\|t f\| & =\|t f\|_{\infty}+\left(\int_{\Omega}\left(d d^{c} u(t f)\right)^{n}\right)^{\frac{1}{n}}=|t|\|f\|_{\infty}+|t|\left(\int_{\Omega}\left(d d^{c} u(f)\right)^{n}\right)^{\frac{1}{n}} \\
& =|t|\|f\| .
\end{aligned}
$$

Thus $(\mathscr{C} \mathscr{P}(\partial \Omega),\|\cdot\|)$ is a normed vector space, since the triangle inequality follows from Lemma 2.3. It remains to prove completeness. Now assume that $\left[f_{j}\right]$ is a Cauchy sequence in $(\mathscr{C} \mathscr{P}(\partial \Omega),\|\cdot\|)$, hence it is a Cauchy sequence in $\|\cdot\|_{\infty}$ norm and therefore there exists a continuous function $f: \partial \Omega \rightarrow \mathbf{R}$ such that $\left[f_{j}\right]$ converges uniformly on $\partial \Omega$ to $f$ as $j \rightarrow+\infty$. Because $\left[f_{j}\right]$ is 
a Cauchy sequence in $(\mathscr{C} \mathscr{P}(\partial \Omega),\|\cdot\|)$ there exists an increasing sequence $j_{k}$ such that $\left\|f_{j_{k+1}}-f_{j_{k}}\right\| \leq 2^{-k}$, hence

$$
\int_{\Omega}\left(d d^{c} u\left(f_{j_{k+1}}-f_{j_{k}}\right)\right)^{n} \leq 2^{-n k},
$$

by (2). Note that

$$
f_{j_{k+1}}=f_{j_{1}}+\sum_{i=1}^{k}\left(f_{j_{i+1}}-f_{j_{i}}\right)
$$

and then by using (17) together with Lemma 2.3 it follows that

$$
\begin{aligned}
\left(\int_{\Omega}\left(d d^{c} u\left(f_{j_{k+1}}\right)\right)^{n}\right)^{\frac{1}{n}} & \leq\left(\int_{\Omega}\left(d d^{c} u\left(f_{j_{1}}\right)\right)^{n}\right)^{\frac{1}{n}}+\sum_{i=1}^{k}\left(\int_{\Omega}\left(d d^{c} u\left(f_{j_{i+1}}-f_{j_{i}}\right)\right)^{n}\right)^{\frac{1}{n}} \\
& \leq\left\|f_{j_{1}}\right\|+1 .
\end{aligned}
$$

Thus, $\sup _{k} \int_{\Omega}\left(d d^{c} u\left(f_{j_{k+1}}\right)\right)^{n} \leq\left(\left\|f_{1}\right\|+1\right)^{n}<+\infty$. Lemma 3.2 now yields that the total mass of $\left(d d^{c} u(f)\right)^{n}$ is bounded by $\left(\left\|f_{j_{1}}\right\|+1\right)^{n}$, i.e., $f$ satisfies $\mathrm{P} 2$. Now assume that $\mathrm{P} 1$ is false, then there exists a $\xi \in \partial \Omega$ and a sequence $\left[z_{l}\right]$ in $\Omega$ such that $\left[z_{l}\right]$ converges to $\xi$ and $\lim _{l \rightarrow \infty} u\left(z_{l}\right)=-a<0$, where $a \geq 0$ is a constant. The sequence $\left[f_{j}\right]$ converges uniformly to $f$ on $\partial \Omega$, hence there exists $m \in \mathrm{N}$ such that $\left\|f_{j}-f\right\|_{\infty} \leq \frac{a}{4}$ for all $j \geq m$. Lemma 3.2 yields that $\left|u\left(f_{j}\right)\left(z_{l}\right)-u(f)\left(z_{l}\right)\right| \leq 2\left\|f_{j}-f\right\|_{\infty}<\frac{a}{2}$ and a contradiction has been achieved since

$$
\left|\lim _{l \rightarrow \infty}\left(u\left(f_{j}\right)\left(z_{l}\right)-u(f)\left(z_{l}\right)\right)\right|=a .
$$

Thus, $f$ is a compliant function and this proof is completed.

COROLlary 3.4. If $\left[f_{j}\right]$ is a sequence which converges in $(\mathscr{C P}(\partial \Omega),\|\cdot\|)$ to a function $f$, then $\left[\left(d d^{c} u\left(f_{j}\right)\right)^{n}\right]$ converges to $\left(d d^{c} u(f)\right)^{n}$ in the weak ${ }^{*}$ topology. In other words the map $\mathscr{C} \mathscr{P}(\partial \Omega) \ni f \rightarrow\left(d d^{c} u(f)\right)^{n}$ is continuous.

THEOREM 3.5. The set $\mathscr{C} \mathscr{P}_{0}(\partial \Omega)$ is a closed subspace of $\mathscr{C} \mathscr{P}(\partial \Omega)$. Moreover, $\mathscr{C P}_{0}(\partial \Omega)=\mathscr{P} \mathscr{H}(\partial \Omega)$.

Proof. Inequality (16) implies that $\mathscr{C} \mathscr{P}_{0}(\partial \Omega)$ is a subspace. Now assume that $\left[f_{j}\right]$ is a sequence in $\mathscr{C} \mathscr{P}_{0}(\partial \Omega)$ which converges in norm to a function $f$. Corollary 3.4 implies that $\left[\left(d d^{c} u\left(f_{j}\right)\right)^{n}\right]$ converges to $\left(d d^{c} u(f)\right)^{n}$ in the weak* topology. By assumption $u\left(f_{j}\right)=0$, hence $\left(d d^{c} u\left(f_{j}\right)\right)^{n}=0$ and therefore it follows by Corollary 3.4 that $\left(d d^{c} u(f)\right)^{n}=0$. Hence $u(f)=0$, i.e., $f \in$ $\mathscr{C P} \mathscr{P}_{0}(\partial \Omega)$ which yields that $\mathscr{C} \mathscr{P}_{0}(\partial \Omega)$ is closed.

To prove the second statement let $f \in \mathscr{C P P}_{0}(\partial \Omega)$, i.e., $u(f)$ is identically 0 . This together will Walsh's theorem implies that $P B_{f}, P B_{-f} \in \mathscr{P} \mathscr{S} \mathscr{H}(\Omega) \cap$ 
$C(\bar{\Omega})$ and $-P B_{f}=P B_{-f}$. Thus, $P B_{f} \in \mathscr{P} \mathscr{H}(\Omega) \cap C(\bar{\Omega})$. On the other hand, if there exists a function $u \in \mathscr{P} \mathscr{H}(\Omega) \cap C(\bar{\Omega})$ such that $u=f$ on $\partial \Omega$, then $d d^{c} u=0$ and therefore $u=P B_{f}$. Similarly, $-u=P B_{-f}$. Thus, $u(f)$ is identically 0 .

REMARK. It was proved in [7] that if $D$ is a hyperconvex product domain $D=D_{n_{1}} \times \cdots \times D_{n_{k}}, n=n_{1}+\cdots+n_{k}, k \geq 3$, then $\mathscr{C} \mathscr{P}_{0}(\partial D)=\mathscr{C} \mathscr{P}(\partial D)=$ $\mathscr{P} \mathscr{H}(\partial D)$. Consider $f\left(z_{1}, z_{2}\right)=\left|z_{1}\right|^{2}$ defined on the boundary of the unit ball in $C^{2}$. This simple example shows by some straight forward calculations that $\mathscr{C} \mathscr{P}_{0}(\partial B)$ is generally not equal to $\mathscr{C} \mathscr{P}(\partial B)$.

\section{Examples}

Example 4.1 shows that it is not enough to assume that $f$ is a $C^{1}$-function to ensure that $\mathrm{P} 2$ is true.

ExAmple 4.1. Let $B \subseteq \mathrm{C}^{n}$ be the unit ball and $z=\left(z^{\prime}, z_{n}\right) \in B$. For fixed $0<p<1$, let $f_{p}: \partial B \rightarrow \mathrm{R}$ be the function defined by $f_{p}\left(z^{\prime}, z_{n}\right)=\left|z_{n}\right|^{2 p}$. Then

$$
P B_{f_{p}}\left(z^{\prime}, z_{n}\right)=\left|z_{n}\right|^{2 p} \quad \text { and } \quad P B_{-f_{p}}\left(z^{\prime}, z_{n}\right)=-\left(1-\left|z^{\prime}\right|^{2}\right)^{p} .
$$

Set $E=\left\{\left(z^{\prime}, z_{n}\right) \in B: z_{n}=0\right\} \cup\left\{\left(z^{\prime}, z_{n}\right) \in B:\left|z^{\prime}\right|=1\right\}$. The set $E$ is a pluripolar, hence

$$
\begin{aligned}
\int_{\Omega}\left(d d^{c}\left(P B_{f_{p}}+P B_{-f_{p}}\right)\right)^{n} & =\int_{\Omega \backslash E}\left(d d^{c}\left(P B_{f_{p}}+P B_{-f_{p}}\right)\right)^{n} \\
& =C \int_{0}^{1}|r|^{2 n p-2 n+1}\left(1-r^{2}\right)^{n-1} d r
\end{aligned}
$$

where $C>0$ is a constant only depending on $n$ and $p$. Thus $f_{p}$ is not compliant if, and only if, $p \leq \frac{n-1}{n}$. For $n>2$, the function $f_{\frac{n-1}{n}}$ belongs to $C^{1}(\partial B)$.

Example 4.2 shows that even if $\mathrm{P} 2$ is true and $f \in C^{\infty}(\partial \Omega)$, it may happen that $\mathrm{P} 1$ is false. This phenomenon cannot happen if $\Omega$ is a B-regular domain (see e.g. [12] for the definition and elementary properties of B-regular domains).

ExAmple 4.2. Let $P$ be the unit polydisc in $C^{2}$, i.e., $P=\left\{\left(z_{1}, z_{2}\right) \in\right.$ $\left.\mathrm{C}^{2}:\left|z_{1}\right|<1,\left|z_{2}\right|<1\right\}$ and let the function $f: \partial P \rightarrow \mathrm{R}$ be defined by $f\left(z_{1}, z_{2}\right)=\left|z_{2}\right|^{2}$. Then $f \in C^{\infty}(\partial P) P B_{f}\left(z_{1}, z_{2}\right)=\left|z_{2}\right|^{2}$. From (1) it follows that $P B_{-f}\left(z_{1}, z_{2}\right) \geq-1$. Fix $\left(z_{0}, w_{0}\right) \in P$ and let $v_{z_{0}}$ be a function defined on the unit disc $D$ in $C$ by $v_{z_{0}}(\zeta)=P B_{-f}\left(z_{0}, \zeta\right)$, hence $v_{z_{0}} \in \mathscr{S} \mathscr{H}(D)$ and $\lim \sup _{\zeta \rightarrow \xi} v_{z_{0}}(\zeta) \leq-1$ for every $\xi \in \partial D(0,1)$, hence $v_{z_{2}} \leq-1$ on 
$D(0,1)$ by the maximum principle for subharmonic functions and therefore $P B_{-f} \leq-1$ on $P$. Thus, $P B_{-f}\left(z_{1}, z_{2}\right)=-1$. Moreover,

$$
\int_{P}\left(d d^{c}\left(P B_{f}+P B_{-f}\right)\right)^{2}=0 .
$$

Thus P2 is true, but

$$
\lim _{\substack{\left(z_{1}, z_{2}\right) \rightarrow\left(\xi_{1}, \xi_{2}\right) \\\left(z_{1}, z_{2}\right) \in P}}\left(P B_{f}\left(z_{1}, z_{2}\right)+P B_{-f}\left(z_{1}, z_{2}\right)\right) \neq 0,
$$

when $\left(\xi_{1}, \xi_{2}\right) \in \partial P \backslash\left\{\left(w_{1}, w_{2}\right) \in \mathrm{C}^{2}:\left|w_{2}\right|=1\right\}$, hence $\mathrm{P} 1$ is false.

Proposition 4.3. If $D \subseteq \mathrm{C}^{n}$ is a bounded, strictly pseudoconvex domain with $C^{2}$-boundary and $f \in C^{2}(\partial D)$, then $f$ is a compliant function.

Proof. The domain $D$ is in particular B-regular and the function $f$ is in particular continuous and therefore it follows that P1 is true. There exists an open neighbourhood $U$ of $D$ and a strictly plurisubharmonic $C^{2}$-function $\rho: U \rightarrow \mathrm{R}$ such that $\rho=0$ on $\partial \Omega$, since $D$ is a strictly pseudoconvex domain with $C^{2}$-boundary. By Theorem I in [14] there exists a $C^{2}$-function $\tilde{f}: \mathrm{C}^{n} \rightarrow \mathrm{R}$ such that $\tilde{f}=f$ on $\partial D$. Choose $A>0$ such that $u=(\tilde{f}+$ $A \rho) \in \mathscr{P} \mathscr{S} \mathscr{H}(D)$ and $B>0$ such that $v=(-\tilde{f}+B \rho) \in \mathscr{P} \mathscr{S} \mathscr{H}(D)$. Hence, $u, v \in \mathscr{P} \mathscr{S} \mathscr{H}(U) \cap C^{2}(U), u=-v=f$ on $\partial D$. Thus

$$
\int_{D}\left(d d^{c}(u+v)\right)^{n}=\int_{D} \sum_{k=0}^{n}\left(\begin{array}{l}
n \\
k
\end{array}\right)\left(d d^{c} u\right)^{n-k} \wedge\left(d d^{c} v\right)^{k}<+\infty .
$$

The construction of $P B_{f}$ and $P B_{-f}$ implies that $u+v \leq P B_{f}+P B_{-f}$, hence

$$
\int_{D}\left(d d^{c}\left(P B_{f}+P B_{-f}\right)\right)^{n} \leq \int_{D}\left(d d^{c}(u+v)\right)^{n}<+\infty,
$$

by (18) and the comparison principle. Thus P2 holds.

Remark. Let $k \in\{0,1,2, \ldots\} \cup\{+\infty\}$. See [14] for the definition of how a function $f: \partial \Omega \rightarrow \mathrm{R}$ is of class $C^{k}$ and their basic properties.

\section{REFERENCES}

1. Bedford, E., and Federbush, P., Pluriharmonic boundary values, Tôhoku Math. J. 26 (1974), 505-511.

2. Begehr, H., and Dzhuraev, A., An Introduction to Several Complex Variables and Partial Differential Equations, Pitman Monogr. Surveys Pure Appl. Math. 88 (1997).

3. Cegrell, U., Pluricomplex energy, Acta Math. 180 (1998), 187-217. 
4. Cegrell, U., The general definition of the complex Monge-Ampère operator, Ann. Inst. Fourier (Grenoble) 54 (2004), 159-179.

5. Cegrell, U., and Wiklund, J., A Monge-Ampère norm for delta-plurisubharmonic functions, Math. Scand. 97 (2005), 201-216.

6. Czyż, R., and Åhag, P., On the Cegrell classes, IMUJ Preprint 11 (2004). (http://www.im.uj.edu.pl/research/preprints/)

7. Czyż, R., and Åhag, P., Characterization of the compliant functions on hyperconvex product domains, IMUJ Preprint 6 (2005). (http://www.im.uj.edu.pl/research/preprints/)

8. Dzhuraev, A., On Riemann-Hilbert boundary problem in several complex variables, Complex Variables Theory Appl. 29 (1996), 287-303.

9. Perotti, A., Tangential form of the trace condition for pluriharmonic functions in $\mathrm{C}^{n}$, Complex Variables Theory Appl. 48 (2003), 615-623.

10. Perotti, A., Dirichlet problem for pluriharmonic functions of several complex variables, Comm. Partial Differential Equations 24 (1999), 707-717.

11. Rudin, W., Function Theory in the Unit Ball of $\mathrm{C}^{n}$, Grundlehren Math. Wiss. 241 (1980).

12. Sibony, N., Une classe de domaines pseudoconvexes, Duke Math. J. 55 (1987), 299-319.

13. Walsh, J. B., Continuity of envelopes of plurisubharmonic functions, J. Math. Mech. 18 (1968), $143-148$.

14. Whitney, H., Analytic extensions of differentiable functions defined in closed sets, Trans. Amer. Math. Soc. 36 (1934), 63-89.

15. Åhag, P., The complex Monge-Ampère operator on bounded hyperconvex domains, $\mathrm{Ph} . \mathrm{D}$. Thesis, Umeå Univ., 2002.

INSTITUTE OF MATHEMATICS

JAGIELLONIAN UNIVERSITY

REYMONTA 4

30-059 KRAKOW

POLAND

E-mail: Rafal.Czyz@im.uj.edu.pl 